Article

\title{
Graphene Oxide Reinforced Alginate/PVA Double Network Hydrogels for Efficient Dye Removal
}

\author{
Cuiyun Liu ${ }^{1}$, Hongyu Liu ${ }^{1}$ * (D), Tianhui Xiong ${ }^{1}$, Airong Xu ${ }^{1}$, Bingli Pan ${ }^{1}$ and Keyong Tang ${ }^{2, *}$ \\ 1 Chemical Engineering and Pharmaceutics School, Henan University of Science and Technology, \\ Luoyang 471023, China; liucy1218@126.com (C.L.); 18438608207@163.com (T.X.); airongxu@126.com (A.X.); \\ blpan@haust.edu.cn (B.P.) \\ 2 Material Science and Engineering School, Zhengzhou University, Zhengzhou 450001, China \\ * Correspondence: liuhy2003@ustc.edu (H.L.); kytang@zzu.edu.cn (K.T.); Tel.: +86-379-6423-1914 (H.L.)
}

Received: 8 June 2018; Accepted: 25 July 2018; Published: 28 July 2018

\begin{abstract}
Dually crosslinked graphene oxide reinforced alginate/polyvinyl alcohol (PVA) double network (DN) hydrogels were prepared via a facile freeze/thaw method followed by soaking in a $\mathrm{Ca}^{2+}$ solution. The morphology and structure of the hydrogels were systematically examined by Fourier transform infrared spectroscopy (FTIR), X-ray diffraction (XRD), scanning electron microscopy (SEM), and thermogravimetric analysis (TGA). The effects of $\mathrm{pH}$, dosage of hydrogel, adsorption time, and temperature on the adsorptive property of DN hydrogels towards methylene blue (MB) were also studied. Results indicated that the hydrogels exhibited typical 3D porous structures and had an efficient adsorption effect towards MB due to strong interactions between DN hydrogels and MB molecules. The adsorption isotherm was found to coincide with the Langmuir model with a monolayer adsorption. The highest adsorption capacity of DN hydrogels for MB was examined as $480.76 \mathrm{mg} \cdot \mathrm{g}^{-1}$.
\end{abstract}

Keywords: sodium alginate; PVA; graphene oxide; double network hydrogel; adsorption

\section{Introduction}

Water pollution originating from all kinds of organic dyes is on the rise due to the rapid development of printing, dyeing, and chemical industry. On account of containing strongly toxic, highly colored, and poorly biodegradable organic dyes, dyestuff wastewater affects the ecological environment and human health seriously [1-3]. Methylene blue (MB) is a water-soluble azo dye extensively used for dye printing, biological staining, as a chemical indicator, and so on. In order to reduce the pollution of dyes to water, contamination of MB for instance, many sewage treatment technologies, including biological treatment, chemical oxidation, catalytic ozonation, membrane separation, and adsorption have been developed so far [4-6]. Among them, adsorption is a simple, effective, low-cost, and highly available method. However, the exploitation of highly efficient, environmentally friendly adsorbents remains a challenge due to the complexity of wastewater.

In recent years, hydrogels with 3D structures as adsorptive materials for the purpose of dye wastewater treatment have drawn widespread attention [7-9]. Dye molecules can permeate into 3D hydrogels with high adsorption rates, integrating with molecular chains of hydrogels by forming hydrogen bonding or electrostatic interactions; therefore, these dye molecules can be readily immobilized by the hydrogel chains. Consequently, porous hydrogels exhibit high adsorption capacities towards different dyes via their versatile structures and properties. Sodium alginate (SA) is a biological linear natural polymer with the merits of biodegradability, low-cost, and non-toxic properties as well as a wealth of sources. It has been widely studied in wastewater treatment applications because of its preferable adsorption property [10,11]. However, the hydrogels obtained 
from SA exhibit drawbacks such as decreased strength, low superficial area, and weak chemical stability, therefore restricting its application in wastewater treatment. Double network (DN) hydrogels comprising two interpenetrating and cross-linked polymer networks have gained tremendous attention due to their enhanced mechanical property. Many investigations have reported that DN hydrogels exhibited improved mechanical properties and high adsorption capacities for heavy metals and dyes [12-14]. For example, a SA/polyvinyl alcohol (PVA) DN hydrogel was prepared for the purpose of achieving enhanced mechanical properties and electrical conductivity [15]. Moreover, the DN hydrogels can be further reinforced by incorporating inorganic materials, such as graphene sheets.

So far, graphene and its derivates have attracted enormous interest due to their unique properties, such as large special surface area, excellent mechanical properties, and unapproachable barrier properties [16-19]. Compared with hydrophobic graphene, graphene oxide (GO) sheets decorated with a mass of hydroxyl, carboxyl, epoxy, and carboxide groups exhibit good water solubility and benign compatibility with hydrogels [20]. For example, a mechanically robust and printable 3D hydrogel based on alginate and graphene oxide was reported by Liu et al. [21]. However, there have been few studies conducted to examine the influence of graphene sheets on DN hydrogels. Based on these considerations, we herein report a graphene oxide reinforced SA/PVA DN hydrogel for $\mathrm{MB}$ adsorption application via a facile freeze/thaw method followed by soaking in a $\mathrm{Ca}^{2+}$ solution. The construction of SA/PVA DN structures greatly increased the mechanical property of SA hydrogels. Meanwhile, the incorporation of graphene oxide sheets into the hydrogels not only further enhanced the mechanical property, but also affected the morphology of the DN hydrogels, yielding smaller pore size and larger pore density.

\section{Experimental}

\subsection{Materials}

Sodium alginate $\left(M_{\mathrm{W}} \sim 250,000 \mathrm{~g} \cdot \mathrm{mol}^{-1}\right)$, polyvinyl alcohol $\left(M_{\mathrm{W}} \sim 74,800 \mathrm{~g} \cdot \mathrm{mol}^{-1}\right)$, concentrated sulfuric acid $(95 \%)$, hydrochloric acid, potassium permanganate, sodium nitrate, and hydrogen peroxide (30\%) were purchased from Sinopharm Chemical Reagent Co. Ltd., Shanghai, China. Natural flake graphite $(>99 \%)$ was bought from Nanjing Pionerr Nano Co. Ltd., Nanjing, China. Anhydrous calcium chloride was purchased from Tianjin Fengchuan Chemical Reagent Technologies Co. Ltd., Tianjin, China. All materials were directly used without further purification.

\subsection{Fabrication of Graphene Oxide (GO) Reinforced SA/PVA DN Hydrogels}

Graphite oxide was prepared by a modified Hummer's method [22]. The as-prepared brown graphite oxide was purified by washing with huge amounts of deionized (DI) water to remove residual acids. Then, graphite oxide sponge was obtained by freeze drying. Finally, $0.5 \mathrm{~g}$ graphite oxide sponge was ultrasonicated in $500 \mathrm{~mL}$ DI water for $30 \mathrm{~min}$ to get a brown GO solution.

PVA solution was prepared by dissolving 5 g PVA powder in $100 \mathrm{~mL}$ DI water under continuous magnetic stirring at $90^{\circ} \mathrm{C}$. After natural cooling, $5 \mathrm{~g}$ SA powder was added into the PVA solution. After magnetic stirring for $2 \mathrm{~h}$, the uniform mixture was degassed and then poured into a mould. Subsequently, the mixture was placed in a freezer and kept at $-20^{\circ} \mathrm{C}$ for $18 \mathrm{~h}$. Then, the frozen hydrogels were allowed to thaw at room temperature (RT) until the ice melted. This freezing/thawing process was repeated 3 times to form physical crosslinked points between PVA chains. Finally, the hydrogel specimens were dipped into a $4 \mathrm{wt} \% \mathrm{CaCl}_{2}(200 \mathrm{~mL})$ solution for $6 \mathrm{~h}$ to crosslink SA chains, yielding SA/PVA DN hydrogels. Residual $\mathrm{Ca}^{2+}$ within the DN hydrogels was removed by swelling hydrogels in a mass of DI water. GO reinforced SA/PVA DN hydrogels were prepared by the same procedures with loadings of 100, 300, 500, and $700 \mathrm{mg} \mathrm{GO}$, respectively. The as-prepared hydrogels with GO contents of 1, 3, 5, and $7 \mathrm{wt} \%$ were designated as GO1-SA/PVA, GO3-SA/PVA, GO5-SA/PVA, and GO7-SA/PVA, respectively. The preparation procedures and crosslinking mechanisms of the GO reinforced DN hydrogels are schematically represented in Figure 1. 


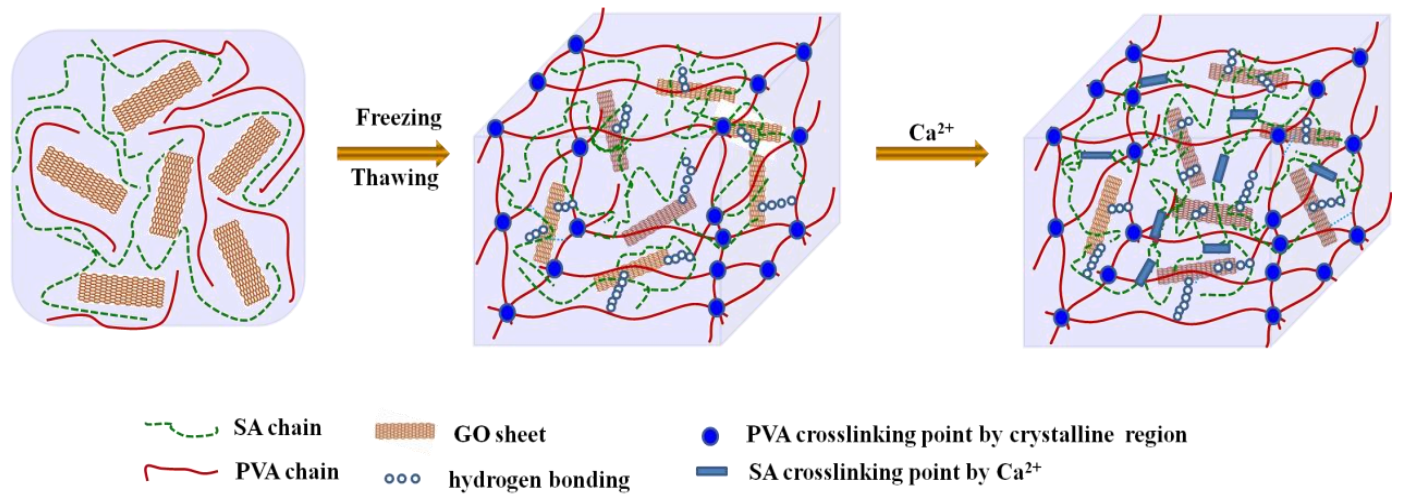

Figure 1. Schematic of crosslinking mechanism of graphene oxide (GO) reinforced double network (DN) hydrogel.

\subsection{Characterizations}

Fourier transform infrared (FTIR) measurement was carried out by using a potassium bromide micropellet technique on a Nicolet iS50 spectrometer (Thermo Fisher Scientific, Waltham, MA, USA). X-ray diffraction (XRD) analysis was performed on a D8 X-ray diffractometer $(40 \mathrm{kV}, 40 \mathrm{~mA}$, $\lambda=0.154 \mathrm{~nm}$, Bruker, Karlsruhe, Germany) by using a powder diffraction method. Thermogravimetric analysis was conducted by a TQ50 from room temperature to $700{ }^{\circ} \mathrm{C}$ at a heating rate of $10{ }^{\circ} \mathrm{C} \cdot \mathrm{min}^{-1}$ in nitrogen flow (TA Instruments, New Castle, DE, USA). Prior to scanning electron microscopy (SEM) study, the fractured freeze-dried hydrogels were coated with a thin layer of gold (JSM-5610LV, Japan Electronics Co. Ltd., Tokyo, Japan). The detection of MB concentration was performed using a UV-visible spectrophotometer (UV5200, Metash instrument Co. Ltd., Shanghai, China). Tensile test experiments were carried out on a CMT6104 electronic-universal testing machine (Shenzhen Skyan Power Equipment Co. Ltd., Shenzhen, China).

\subsection{MB Adsorption Studies}

MB adsorption experiment was performed by shaking a certain mass of GO reinforced DN hydrogels in $25 \mathrm{~mL} \mathrm{MB}$ solution with an agitating speed of $100 \mathrm{rpm}$ at $30^{\circ} \mathrm{C}$ for $15 \mathrm{~h}$ to reach the absorbance equilibrium state. $\mathrm{HCl}$ and $\mathrm{NaOH}$ solutions were used in order to adjust the $\mathrm{pH}$ values of the adsorption solutions. Absorbance of the solution was measured by a UV-visible spectrophotometer under a wavelength of $664 \mathrm{~nm}$. The influences of GO loadings (under $\mathrm{pH} 6.5$ ) and $\mathrm{pH}$ on the adsorption were examined by soaking a $0.025 \mathrm{~g}$ sample into $25 \mathrm{~mL}$ of MB solution $\left(200 \mathrm{mg} \cdot \mathrm{L}^{-1}\right)$ for $15 \mathrm{~h}$. The influence of the initial concentration of $\mathrm{MB}$ on the adsorption was performed by adding $0.025 \mathrm{~g}$ of specimen into $25 \mathrm{~mL}$ of MB solution $\left(50,100,150,200,300,400,500,700\right.$, and $900 \mathrm{mg} \cdot \mathrm{L}^{-1}$, respectively) for $15 \mathrm{~h}(\mathrm{pH}=6.5)$. The effect of contact time on the adsorption was investigated by soaking $0.15 \mathrm{~g}$ of sample into $150 \mathrm{~mL}$ of MB solution under $\mathrm{pH}$ of 6.5 . The adsorption capacity $\left(q_{\mathrm{t}}\right)$ and removal efficiency $(R e)$ were calculated from the following expressions:

$$
\begin{aligned}
q_{\mathrm{t}} & =\frac{\left(C_{0}-C_{\mathrm{t}}\right) V}{m} \\
\operatorname{Re}(\%) & =\frac{C_{0}-C_{\mathrm{t}}}{C_{0}} \times 100 \%
\end{aligned}
$$

where $q_{\mathrm{t}}$ is the adsorption capacity $\left(\mathrm{mg} \cdot \mathrm{g}^{-1}\right.$ ) and $C_{0}$ is the initial MB concentration, whereas $C_{\mathrm{t}}$ is the MB concentration at any time, $t\left(\mathrm{mg} \cdot \mathrm{L}^{-1}\right) . V$ was the volume of the experimental solution $(\mathrm{mL})$ and $m$ was the mass of adsorbent $(\mathrm{g})$.

In order to investigate the adsorption process and adsorption mechanism of GO reinforced DN hydrogels, three kinds of adsorption kinetic equations, namely, the pseudo-first-order equation, 
pseudo-second-order equation, and intraparticle diffusion equation, were used to fit the experimental data [23].

The pseudo-first-order kinetic model is expressed as:

$$
\ln \left(q_{\mathrm{e}}-q_{\mathrm{t}}\right)=\ln q_{\mathrm{e}}-k_{1} t
$$

where $q_{\mathrm{t}}$ and $q_{\mathrm{e}}$ stand for the adsorption capacities of DN hydrogels $\left(\mathrm{mg} \cdot \mathrm{g}^{-1}\right)$ at $t$ and the equilibrium state, respectively. $k_{1}\left(\mathrm{~min}^{-1}\right)$ is the rate constant.

The pseudo-second-order equation is expressed as:

$$
\frac{t}{q_{\mathrm{t}}}=\frac{1}{k_{2} q_{\mathrm{e}}^{2}}+\frac{t}{q_{\mathrm{e}}}
$$

where $k_{2}\left(\mathrm{~g} \cdot \mathrm{mg}^{-1} \cdot \mathrm{min}^{-1}\right)$ is the pseudo-second-order adsorption rate constant.

The intraparticle diffusion model is expressed as:

$$
q_{\mathrm{t}}=k_{\mathrm{i}} t^{\frac{1}{2}}+C
$$

where $k_{\mathrm{i}}$ is the rate constant of the intraparticle diffusion model $\left(\mathrm{mg} \cdot \mathrm{g}^{-1} \cdot \mathrm{min}^{-1 / 2}\right)$ and $C$ is a constant standing for the boundary layer effects.

\section{Results and Discussion}

\subsection{Formation Mechanism of GO Reinforced SA/PVA DN Hydrogels}

The dual crosslinking mechanisms of GO reinforced SA/PVA DN hydrogels were schematically presented in Figure 1. As could be seen, PVA chains were crosslinked by forming crystalline regions during the freezing/thawing treatments of PVA solution. On the other hand, alginate chains were crosslinked by $\mathrm{Ca}^{2+}$ ions, as described elsewhere [24]. Furthermore, the presence of GO offered hydrogen bonding interaction between GO sheets and PVA/SA chains. Therefore, GO sheets worked as additional crosslinking points in SA/PVA DN hydrogels. This conclusion was further confirmed by FTIR and SEM studies.

\subsection{FTIR Analysis}

FTIR spectrometry was employed for monitoring the interactions between GO sheets and polymer networks. As shown in Figure 2, the FTIR spectrum of GO exhibited characteristic peaks of $-\mathrm{OH}, \mathrm{C}=\mathrm{O}$, $\mathrm{C}=\mathrm{C}$, and $\mathrm{C}-\mathrm{O}-\mathrm{C}$ at $3420,1731,1633$, and $1237 \mathrm{~cm}^{-1}$, respectively. For neat SA/PVA, the predominant peaks located at 3348 and 2922 are ascribed to the stretching vibrations of $-\mathrm{OH}$ and $-\mathrm{CH}_{2}$, respectively. The peaks at 1608 and $1438 \mathrm{~cm}^{-1}$ are attributed to the asymmetric and symmetric stretching vibrations of carboxylate ions, respectively [25]. The characteristic peak located at $1093 \mathrm{~cm}^{-1}$ was due to the stretching vibration of $\mathrm{C}-\mathrm{O}-\mathrm{C}$. In comparison with neat SA/PVA, GO reinforced SA/PVA DN hydrogels exhibited lower wave numbers, possibly due to the dissociation of hydrogen bonding among the hydroxyl groups in PVA and formation of new hydrogen bonding between the hydroxyl groups of PVA/SA with hydroxyl groups of GO sheets [18].

\subsection{Thermogravimetric Analysis}

TGA (TA Instruments, New Castle, DE, USA) and corresponding differential thermogravimetric analysis (DTG) curves are shown in Figure 3a,b, respectively. TGA thermograms exhibited the good thermal stability of specimens below $250{ }^{\circ} \mathrm{C}$. This slight weight loss $(8 \%)$ was attributed to the dissociation of water [19]. Sharp mass loss was observed around $265-340{ }^{\circ} \mathrm{C}$ due to the decomposition of polymer networks. It was noted that the weight loss of DN hydrogels with different GO loadings differed greatly. GO3-SA/PVA exhibited a weight loss of 52\%, whereas GO1-SA/PVA exhibited 
a weight loss of $48 \%$. However, the introduction of GO into SA/PVA DN hydrogels did not improve its thermal stability much.

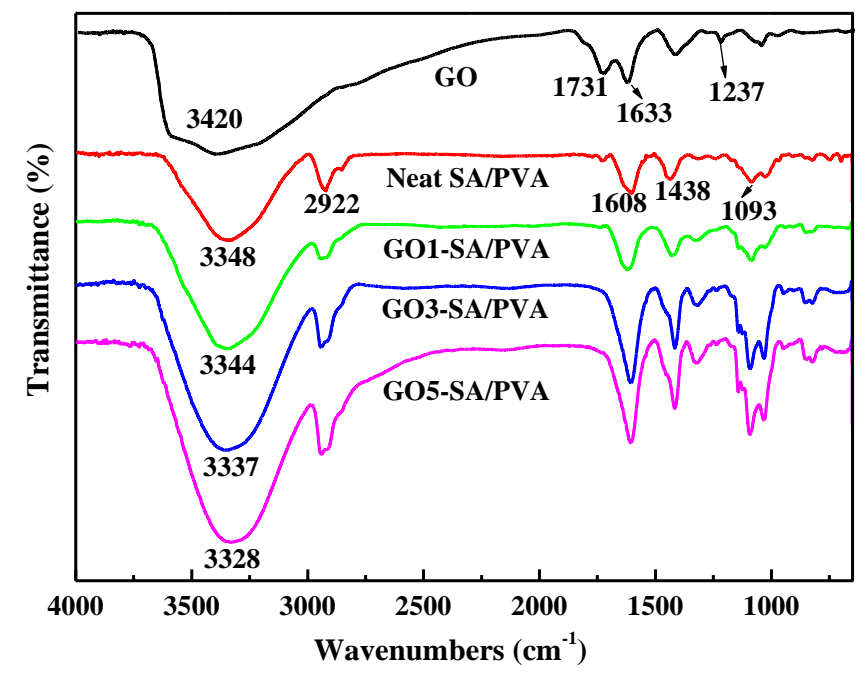

Figure 2. FTIR spectra of GO, DN hydrogel, and DN hydrogels with different GO loadings.
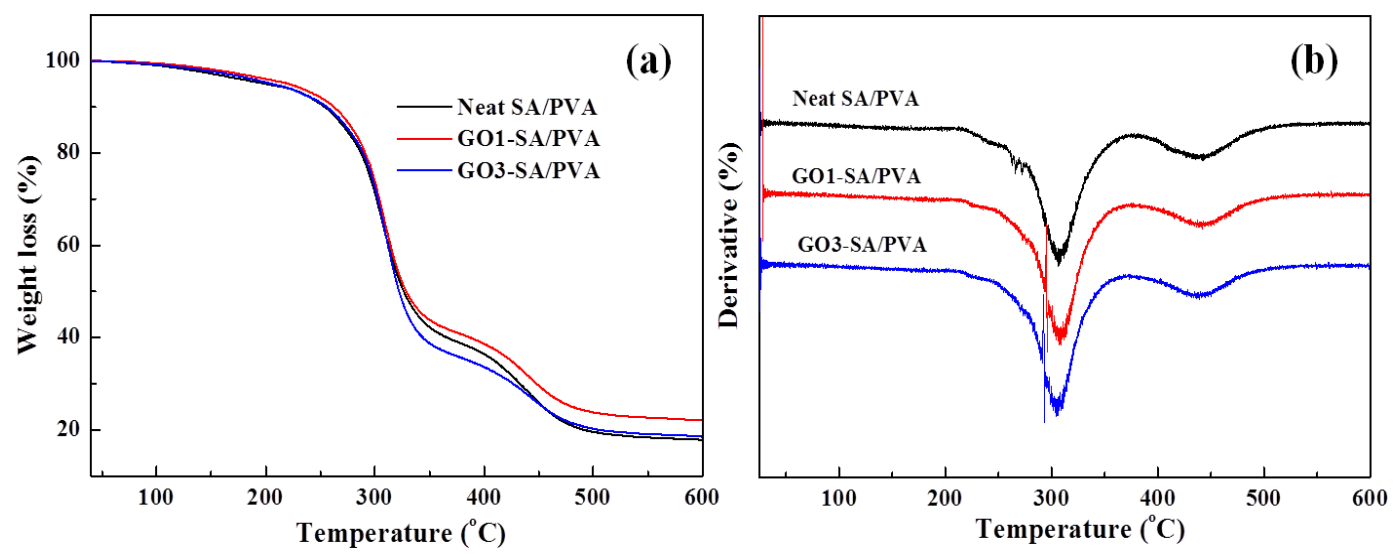

Figure 3. (a) TGA and (b) differential thermogravimetric analysis (DTG) curves of DN hydrogel and GO reinforced DN hydrogels composites.

\subsection{XRD Analysis}

Figure 4 shows the XRD patterns of GO and GO reinforced SA/PVA specimens. As was shown, the XRD pattern of GO exhibited a sharp peak at $2 \theta=19.5^{\circ}$. In the case of neat SA/PVA, the peak located at $2 \theta=11.9^{\circ}$ was due to the amorphous diffraction peak of SA. The peaks located at $2 \theta=32^{\circ}$, $34^{\circ}$, and $45^{\circ}$ were due to the presence of excrescent calcium ions in SA [26]. The sharp peak presented at $2 \theta=20.2^{\circ}$ was ascribed to the diffraction peak of PVA [18]. Interestingly, with increasing contents of GO, the intensity of the PVA peak increased slightly, followed by decreasing greatly. The changes in peak intensity demonstrated the corresponding changes in the degree of crystallinity of the SA/PVA hydrogels. It had been reported that the introduction of GO could affect the degree of crystallinity of PVA [18]. The largest degree of crystallinity of PVA in GO1-SA/PVA indicated that the PVA chains in GO1-SA/PVA exhibited the biggest physical crosslinking density by forming crystalline regions. Therefore, it was reasonable to observe the variation tendency in the XRD diffraction peaks of GO reinforced DN SA/PVA hydrogels. The reason was that the addition of nanofiller GO could impact on the crystallization behavior of crystalline polymers due to the heterogeneous nucleation of GO sheets. On the other hand, GO1-SA/PVA also exhibited the highest peaks at $2 \theta=32^{\circ}$ and $34^{\circ}$, in comparison 
to other diffraction peaks. This might be due to the relatively higher amount of residual calcium ions inside the GO1-SA/PVA.

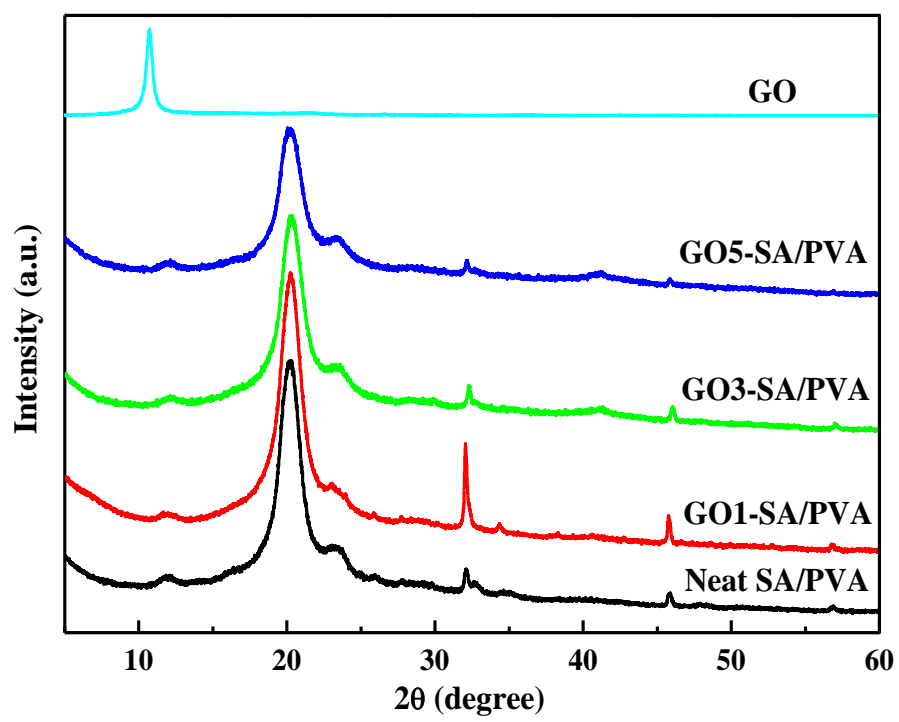

Figure 4. XRD patterns of GO, neat DN hydrogel, and GO reinforced DN hydrogels.

\subsection{SEM Characterization}

For further investigating the influence of GO sheets on the morphology of SA/PVA DN hydrogels, samples with different GO loadings were freeze-dried, then coated with a thin layer of gold and subjected to SEM examinations. The results were shown in Figure 5. It could be observed that all SA/PVA DN hydrogels exhibited a porous 3D structure, consistent with previous reports $[27,28]$. However, with increasing GO contents, the SA/PVA composites exhibited smaller pore size, demonstrating the formation of an improved degree of crosslinking. The crosslinking of SA/PVA by GO sheets via hydrogen bonding interactions accounted for this improved degree of crosslinking. Moreover, this increased degree of crosslinking could also enhance the mechanical property of SA/PVA DN hydrogels.
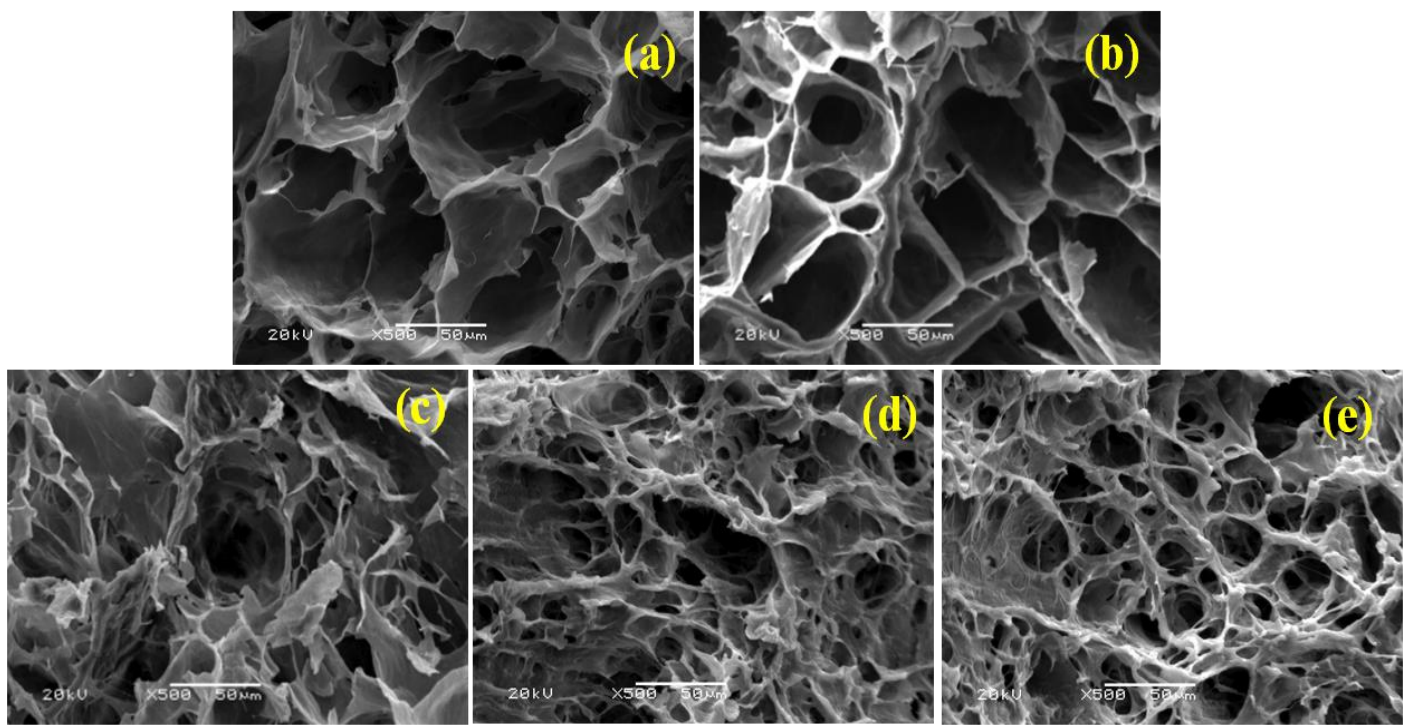

Figure 5. SEM images of SA/PVA with different GO loadings: (a) neat SA/PVA; (b) GO1-SA/PVA; (c) GO3-SA/PVA; (d) GO5-SA/PVA; (e) GO7-SA/PVA. 


\subsection{Mechanical Properties}

The stress-strain curves of the SA/PVA DN hydrogels with different GO loadings are shown in Figure 6. It is known that the degree of crystallinity, degree of crosslinking, and nanofiller dosage can influence the mechanical properties of composites greatly. As depicted, with increasing GO amounts, the SA/PVA DN hydrogels exhibited a tendency to decrease after an increase in degree of crystallinity. On the other hand, the degree of crosslinking was always increased with increasing GO contents. On account of the enhancements by GO sheets and degree of crosslinking, the fracture strength of GO reinforced SA/PVA hydrogels increased from $0.11 \mathrm{MPa}$ for neat SA/PVA to 0.24 MPa for GO7-SA/PVA. In addition, it is worthy to note that the elongation at break of the composite hydrogels increased first, followed by decreasing. The increase in elongation at break for SA/PVA DN hydrogels with a small amount of GO can be ascribed to the formation of hydrogen bonding interaction between GO and polymer chains, as described by Fan et al. [29]. However, excessive embedment of GO sheets will restrict the elasticity of the polymer chains, therefore resulting in a reduction in elongation at break [30].

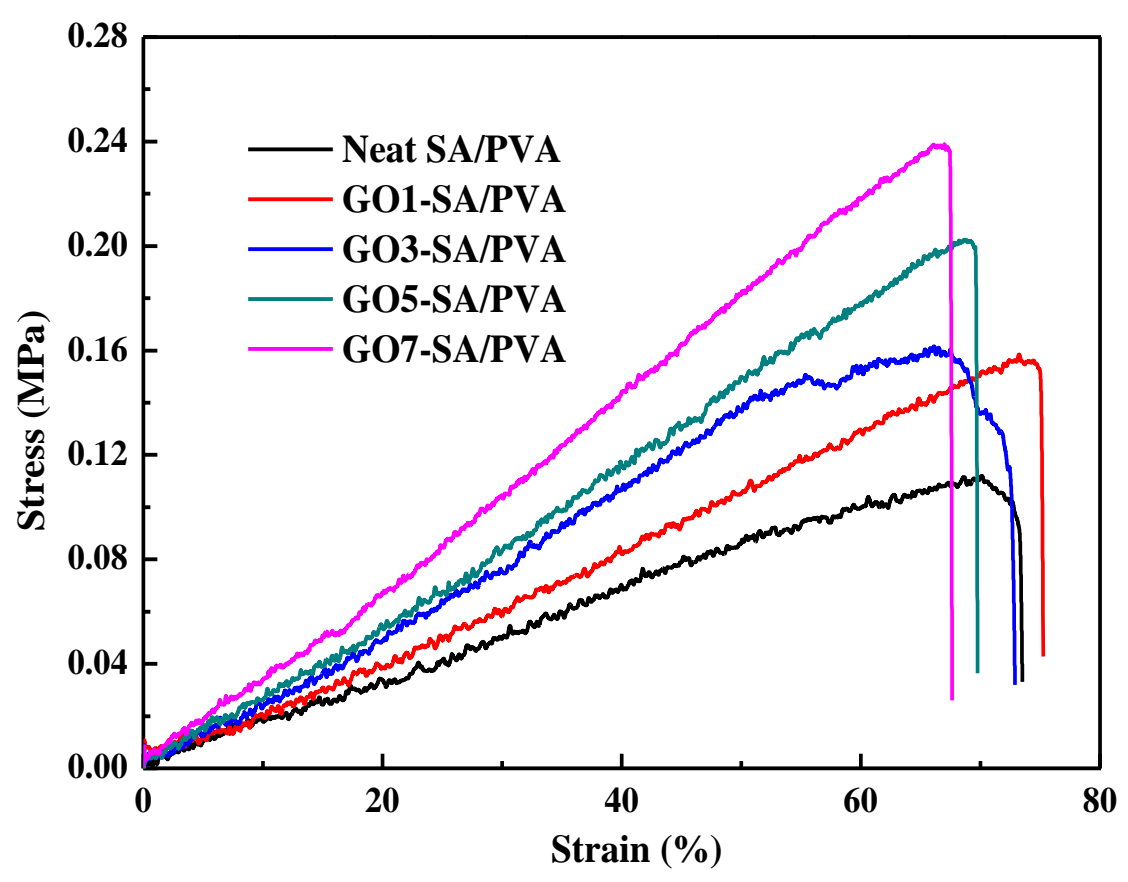

Figure 6. Stress-strain curves of neat DN hydrogel and GO reinforced DN hydrogels.

\subsection{MB Adsorption}

As described previously in Figure 5, GO content affects the morphology of the GO reinforced DN hydrogels greatly. With loading more GO, the composite hydrogels exhibit smaller pore size and higher porosity, therefore affecting the adsorption behavior of MB. The influence of GO content on the adsorption of DN hydrogels for MB is shown in Figure 7a. It can be observed that increased GO content results in an improved adsorption capacity for MB. First, higher GO content results in higher porosity and larger specific area, which can obviously improve the adsorption capacity. Second, GO sheets were functionalized with a vast number of hydroxyl and carboxyl groups. These versatile groups can interact with MB molecules by electrostatic or hydrogen bonding interactions. Finally, GO and MB molecules can have strong $\pi-\pi$ interactions. Based on these factors, improving GO content produces enhanced adsorption capacity. However, when the GO content is higher than $5 \%$, the increase tendency in adsorption capacity is slowed down, probably due to the aggregation of GO sheets. 

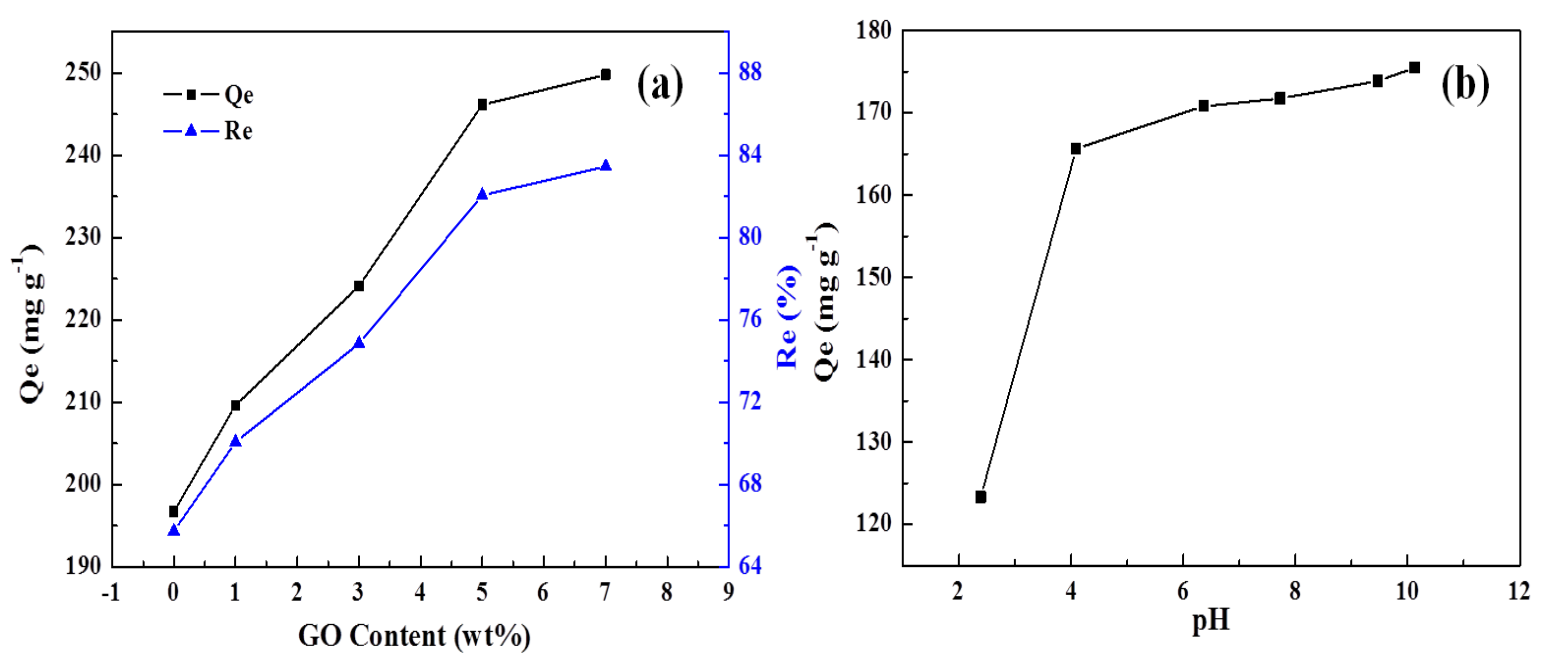

Figure 7. Effects of (a) GO content and (b) solution $\mathrm{pH}$ on the methylene blue (MB) adsorption.

$\mathrm{pH}$ is one of the important factors which influence the adsorption properties of DN hydrogel for MB. The effect of $\mathrm{pH}$ on the $\mathrm{MB}$ adsorption is shown in Figure $7 \mathrm{~b}$. As can be seen, with increasing the $\mathrm{pH}$ values of the solution from 2.4 to 6.4 , the adsorption capacity increases greatly from 123.40 to $165.51 \mathrm{mg} \cdot \mathrm{g}^{-1}$. On the other hand, when further increasing the $\mathrm{pH}$ values from 6.4 to 9.5 , the adsorption capacity does not change much. Due to the presence of versatile oxygen-containing groups decorated in DN hydrogels, such as hydroxyl and carboxyl groups, $\mathrm{MB}$ can be adsorbed onto the DN hydrogels by either electrostatic interaction or hydrogen bonding interaction. Different $\mathrm{pH}$ values not only influence the charge distribution of DN hydrogels, but also the ionization of DN hydrogels and MB. Therefore, the adsorption behavior of $\mathrm{DN}$ hydrogels towards $\mathrm{MB}$ was greatly influenced by $\mathrm{pH}$ values. At low $\mathrm{pH}$ values, the carboxyl groups of SA and GO were protonated. Consequently, it reduces the electrostatic interactions between the DN hydrogels and MB molecules. In addition, excess proton ions will transform the hydroxyl groups of DN hydrogels into $-\mathrm{OH}_{2}{ }^{+}$groups. Then, the electrostatic repulsion between $-\mathrm{OH}_{2}{ }^{+}$groups and cationic dye $\mathrm{MB}$ further reduces the adsorption capacity [31,32]. Hence, at low $\mathrm{pH}$ values, the $\mathrm{DN}$ hydrogels exhibit extremely low adsorption capacities. When increasing $\mathrm{pH}$ values, the carboxyl groups of DN hydrogels were unprotonated as anions; the electrostatic interaction between the carboxylate radical and $\mathrm{MB}$ molecules accounts for the increased adsorption capacities for MB. The optimum $\mathrm{pH}$ was determined to be 6.4 for $\mathrm{MB}$ [33].

The effect of DN hydrogel dosage on the adsorption was examined by soaking different amounts of hydrogels into $25 \mathrm{~mL}$ of $\mathrm{MB}$ solution with a concentration of $200 \mathrm{mg} \cdot \mathrm{L}^{-1}$. As can be seen from Figure $8 \mathrm{a}$, the equilibrium adsorption capacity of DN hydrogels for $\mathrm{MB}$ was decreased with increasing the dosage of DN hydrogels. When the dosages of DN hydrogel were changed from 0.5 to $8 \mathrm{~g} \cdot \mathrm{L}^{-1}$, the equilibrium adsorption capacities were reduced from 313.09 to $23.09 \mathrm{mg} \cdot \mathrm{g}^{-1}$. For a certain amount of MB solution, DN hydrogels with lowered dosages would readily reach a saturation adsorption state due to the limited adsorption active points in DN hydrogels [34]. However, when increasing the dosage of DN hydrogels, the adsorption active points were in an unsaturated adsorption state, resulting in lowered adsorption capacities. The optimum adsorbent dosage was determined to be $1.00 \mathrm{~g} \cdot \mathrm{L}^{-1}$ for $\mathrm{MB}$.

The influence of contact time on the adsorption was shown in Figure 8b. As can be seen, DN hydrogels exhibit similar adsorption behavior for $\mathrm{MB}$ solutions with different concentrations. At the beginning of the adsorption, the adsorption capacity increases with increasing the contact time. This might be due to the large content of $\mathrm{MB}$ at the initial state, resulting in convenience for the $\mathrm{MB}$ to diffuse into the DN hydrogels. Upon further increasing contact time, the number of MB molecules in solution reduced and the active adsorption points in DN hydrogels were occupied by MB molecules, therefore resulting in a moderate increase in adsorption capacity [35]. 

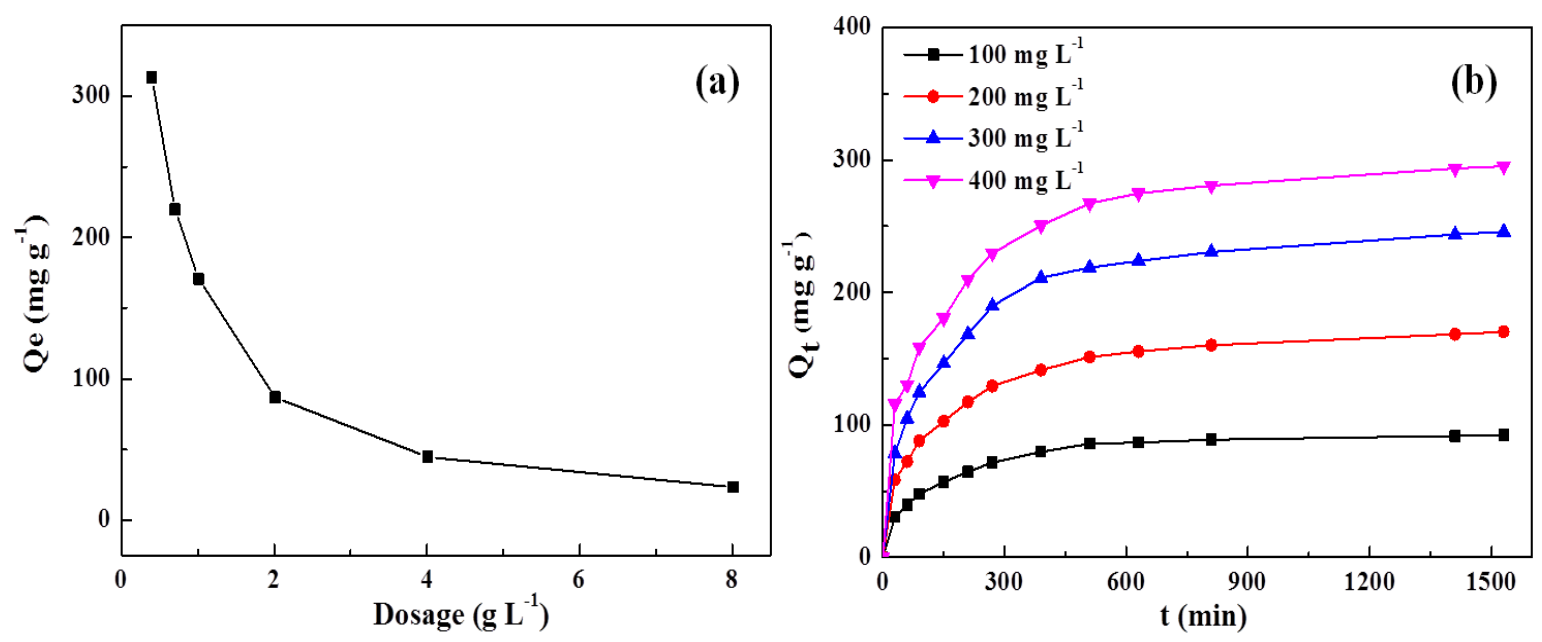

Figure 8. Effects of (a) dosage of adsorbent and (b) contact time on the MB adsorption.

The corresponding kinetic parameters and determination coefficients obtained from the slope and intercept according to these equations are shown in Tables 1 and 2.

In comparison with the pseudo-first-order model, the theoretical equilibrium adsorption capacities of the pseudo-second-order model shown in Figure $9 \mathrm{~b}$ and Table 1 are closer to the experimental values. In addition, the correlation coefficients $\left(R^{2}\right)$ of the pseudo-second-order model are larger than that of the pseudo-first-order model, indicating that the pseudo-second-order model was applicable to the MB adsorption process [32]. Furthermore, the theoretical adsorption capacities of MB increase with increasing $\mathrm{MB}$ concentration, whereas the adsorption rate constant of the pseudo-second-order model decreases with increasing $\mathrm{MB}$ concentration due to the hindrance of higher concentrations of MB. After disclosing the adsorption process, the adsorption mechanism was further discussed by using the intraparticle diffusion model.

Table 1. Adsorption kinetic parameters of MB by adsorbents.

\begin{tabular}{|c|c|c|c|c|c|c|c|}
\hline \multirow{2}{*}{$C_{0}\left(\mathrm{mg} \cdot \mathrm{L}^{-1}\right)$} & \multicolumn{4}{|c|}{ Pseudo-first-order model } & \multicolumn{3}{|c|}{ Pseudo-second-order model } \\
\hline & $q_{\mathrm{e}}(\exp )\left(\mathrm{mg} \cdot \mathrm{g}^{-1}\right)$ & $k_{1}\left(\min ^{-1}\right)$ & $q_{\mathrm{e}}(\mathrm{cal})\left(\mathrm{mg} \cdot \mathrm{g}^{-1}\right)$ & $R^{2}$ & $k_{2}\left(\mathrm{~g} \cdot \mathrm{mg}^{-1} \cdot \mathrm{min}^{-1}\right)$ & $q_{\mathrm{e}}(\mathrm{cal})\left(\mathrm{mg} \cdot \mathrm{g}^{-1}\right)$ & $R^{2}$ \\
\hline 100 & 92.01 & 0.0037 & 59.77 & 0.9912 & $1.14 \times 10^{-4}$ & 97.66 & 0.9985 \\
\hline 200 & 170.12 & 0.0029 & 102.78 & 0.9899 & $5.72 \times 10^{-5}$ & 179.86 & 0.9981 \\
\hline 300 & 245.75 & 0.0031 & 150.61 & 0.9845 & $3.93 \times 10^{-5}$ & 260.42 & 0.9977 \\
\hline 400 & 295.15 & 0.0034 & 181.75 & 0.9936 & $3.67 \times 10^{-5}$ & 311.53 & 0.9982 \\
\hline
\end{tabular}

Table 2. Parameters of intra-particle diffusion model for MB adsorption.

\begin{tabular}{|c|c|c|c|c|c|c|}
\hline \multirow{2}{*}{$C_{0}\left(\mathrm{mg} \cdot \mathrm{L}^{-1}\right)$} & \multicolumn{3}{|c|}{ Step I } & \multicolumn{3}{|c|}{ Step II } \\
\hline & $K_{\mathrm{i} 1}\left(\mathrm{mg} \cdot \mathrm{g}^{-1} \cdot \mathrm{min}^{-1 / 2}\right)$ & $C\left(\mathrm{mg} \cdot \mathrm{g}^{-1}\right)$ & $R^{2}$ & $K_{\mathrm{i} 2}\left(\mathrm{mg} \cdot \mathrm{g}^{-1} \cdot \min ^{-1 / 2}\right)$ & $C\left(\mathrm{mg} \cdot \mathrm{g}^{-1}\right)$ & $R^{2}$ \\
\hline 100 & 3.49 & 13.06 & 0.9899 & 0.39 & 77.04 & 0.9819 \\
\hline 200 & 5.97 & 28.21 & 0.9868 & 1.09 & 127.98 & 0.9825 \\
\hline 300 & 9.31 & 32.32 & 0.9924 & 1.61 & 183.07 & 0.9929 \\
\hline 400 & 9.70 & 60.10 & 0.9867 & 1.61 & 233.05 & 0.9773 \\
\hline
\end{tabular}

According to Figure 9c, all plots don't pass through the origin, indicating that intraparticle diffusion was not the sole rate-controlling step. However, the majority of the correlation coefficients in Table 2 were larger than 0.98 , demonstrating the adsorption mechanism can be explained by the intra-particle diffusion model. There are two stages in the adsorption curves of MB by DN hydrogels exhibiting different slopes. In the first stage, MB molecules diffuse from the solution into the surface 
of the DN hydrogels through large pores or channels inside the porous DN hydrogels. In the second stage, MB molecules diffuse into smaller pores and finally reach the equilibrium state [36].
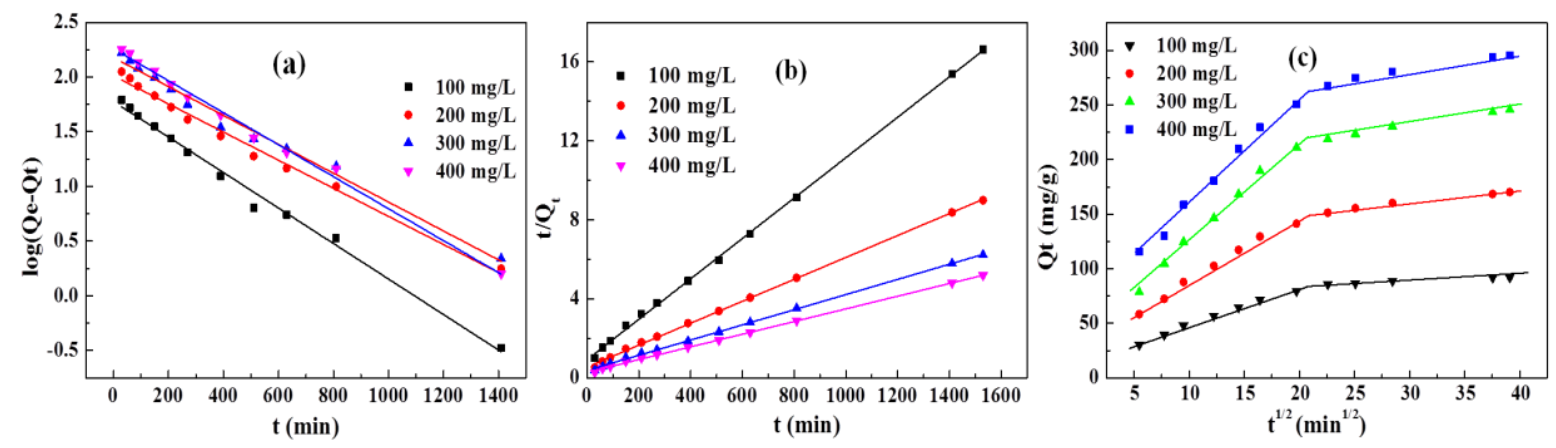

Figure 9. (a) Pseudo-first-order plots, (b) pseudo-second-order plots, and (c) intraparticle diffusion plots for MB adsorption.

Langmuir and Freundlich adsorption isothermal equations are widely used to describe the chemical adsorption behaviours [37]. Langmuir adsorption isotherm relies on the monolayer adsorption and the adsorption points being distributed uniformly among the surface of the adsorbent. The interaction between adsorbent and adsorbate is assumed to be strong enough. The Langmuir adsorption isotherm is expressed as:

$$
\frac{C_{\mathrm{e}}}{q_{\mathrm{e}}}=\frac{1}{q_{\mathrm{m}} K_{\mathrm{L}}}+\frac{C_{\mathrm{e}}}{q_{\mathrm{m}}}
$$

where $q_{\mathrm{e}}$ and $c_{\mathrm{e}}$ are the equilibrium adsorption capacity $\left(\mathrm{mg} \cdot \mathrm{g}^{-1}\right)$ and equilibrium concentration $\left(\mathrm{mg} \cdot \mathrm{g}^{-1}\right)$, respectively. $q_{\mathrm{m}}$ is the maximum adsorption capacity $\left(\mathrm{mg} \cdot \mathrm{g}^{-1}\right)$ and $K_{\mathrm{L}}$ is the rate constant of Langmuir adsorption isotherm.

Freundlich adsorption isothermal is an empirical equation; normally used for elucidating a nonideal adsorption behaviour of adsorbent, it can be expressed as:

$$
\log q \mathrm{e}=\log K_{\mathrm{F}}+\frac{1}{n} \log C_{\mathrm{e}}
$$

where $K_{\mathrm{F}}$ is the rate constant of the Freundlich adsorption isotherm. $1 / n$ is a constant related to adsorption capacity. According to Table 3 , the $1 / n$ value is 0.44 , indicating that MB is easily adsorbed onto DN hydrogels by a Freundlich adsorption model.

Table 3. Adsorption isotherm parameters of Langmuir and Freundlich models.

\begin{tabular}{cccccc}
\hline \multicolumn{3}{c}{ Langmuir model } & \multicolumn{3}{c}{ Freundlich model } \\
\hline $\boldsymbol{q}_{\mathbf{m}}\left(\mathbf{m g} \cdot \mathbf{g}^{-\mathbf{1}}\right)$ & $\boldsymbol{K}_{\mathrm{L}}\left(\mathbf{L} \cdot \mathbf{m g}^{-\mathbf{1}}\right)$ & $\boldsymbol{R}^{\mathbf{2}}$ & $\boldsymbol{K}_{\mathrm{F}}$ & $\mathbf{1} / \boldsymbol{n}$ & $\boldsymbol{R}^{\mathbf{2}}$ \\
\hline 480.76 & 0.02 & 0.9896 & 34.11 & 0.44 & 0.9635 \\
\hline
\end{tabular}

The experimental data were fitted with the Langmuir and Freundlich adsorption isothermal equations, respectively, and the results are shown in Figure 10 and Table 3. The correlation coefficient $\left(R^{2}\right)$ of the Langmuir adsorption model is 0.9896 , larger than that of the Freundlich adsorption model (0.9635), demonstrating that the Langmuir adsorption model is more in accord with the experimental data in comparison with the Freundlich adsorption model.

In order to investigate the influence of temperature on the adsorption process, an adsorption experiment was performed at 298, 303, 313, and $323 \mathrm{~K}$, respectively. Thermodynamic parameters, such as $\Delta G, \Delta H$, and $\Delta S$ were determined as:

$$
K_{\mathrm{c}}=\frac{q_{\mathrm{e}}}{C_{\mathrm{e}}}
$$




$$
\begin{gathered}
\ln K_{\mathrm{c}}=\frac{\Delta S}{R}-\frac{\Delta H}{R T} \\
\Delta G=\Delta H-T \Delta S
\end{gathered}
$$

where $T(\mathrm{~K})$ is temperature and $\Delta H\left(\mathrm{~kJ} \cdot \mathrm{mol}^{-1}\right), \Delta S\left(\mathrm{~J} \mathrm{~mol}^{-1} \mathrm{~K}^{-1}\right)$, and $\Delta G\left(\mathrm{~kJ} \cdot \mathrm{mol}^{-1}\right)$ are the changes in enthalpy, entropy, and Gibbs free energy, respectively.
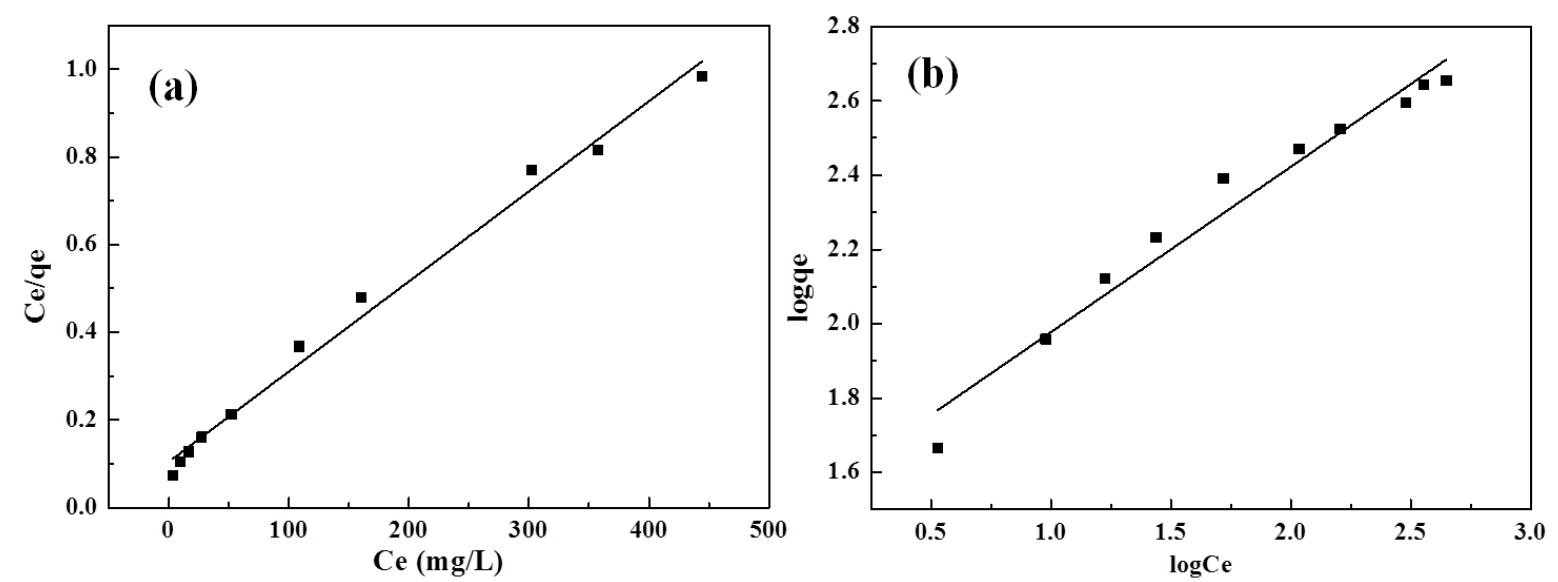

Figure 10. Langmuir (a) and Freundlich (b) adsorption isotherm models of MB.

The results are given in Table 4. As is shown, all $\Delta G$ values are negative, indicating that the adsorption of $\mathrm{MB}$ is a spontaneous process. Furthermore, the value of $\Delta G$ increases with improving temperature, demonstrating that low temperature is favourable for $\mathrm{MB}$ adsorption. $\Delta H$ is negative, demonstrating that the adsorption is an exothermic process. Due to the adsorption of MB molecules onto the surface of DN hydrogels reducing the disorder, the values of $\Delta S$ also are negative. This is in line with a previous report [38].

\begin{tabular}{|c|c|c|c|c|}
\hline$T(\mathrm{~K})$ & $K_{\mathrm{c}}\left(\mathrm{L} \cdot \mathrm{mg}^{-1}\right)$ & $\Delta G\left(\mathrm{~kJ} \cdot \mathrm{mol}^{-1}\right)$ & $\Delta H\left(\mathrm{~kJ} \cdot \mathrm{mol}^{-1}\right)$ & $\Delta S\left(\mathrm{~J} \cdot \mathrm{mol}^{-1} \cdot \mathrm{K}^{-1}\right)$ \\
\hline 298 & 6.94 & -4.80 & \multirow{4}{*}{-15.45} & \multirow{4}{*}{-35.95} \\
\hline 303 & 5.96 & -4.49 & & \\
\hline 313 & 4.90 & -4.14 & & \\
\hline 323 & 4.25 & -3.89 & & \\
\hline
\end{tabular}

Table 4. Thermodynamic parameters for MB adsorption at different temperatures.

\section{Conclusions}

In summary, dually crosslinked SA/PVA DN hydrogels reinforced by GO sheets were successfully prepared via a facile freeze/thaw process followed by soaking in a $\mathrm{Ca}^{2+}$ solution. The as-resulted hydrogels exhibited typical 3D porous structures. Contributed by the fascinating GO sheets, the porous hydrogels exhibited enhanced mechanical properties and excellent adsorption behaviors towards MB. Therefore, the GO reinforced SA/PVA DN hydrogels can be used as a nontoxic, biodegradable, low-cost dye adsorbent to treat dyestuff wastewater. The experimental adsorptive data coincided with the Langmuir model. The pseudo-second-order model could describe the adsorption behaviour of the DN hydrogel for MB well. Thermodynamic investigations elucidated that the adsorption of MB by DN hydrogels was a spontaneous and exothermic process.

Author Contributions: C.L. and H.L. designed the experiments and wrote the paper. T.X. prepared the samples. A.X. and B.P. analyzed the data. K.T. contributed to the reagents/materials.

Funding: We gratefully acknowledge the financial supports by research project of HAUST (No. 13480051), National Natural Science Foundation of China (51675162, 21373078, U1704144), Science and Technology Innovation 
Team Training and Development Plan of HAUST (2015XTD008), Henan Province Science and Technology Research Plan (152102210274).

Conflicts of Interest: The authors declare no conflict of interest.

\section{References}

1. Shahabuddin, S.; Sarih, N.M.; Kamboh, M.A.; Nodeh, H.R.; Mohamad, S. Synthesis of Polyaniline-Coated Graphene Oxide@SrTiO 3 Nanocube Nanocomposites for Enhanced Removal of Carcinogenic Dyes from Aqueous Solution. Polymers 2016, 8, 305. [CrossRef]

2. Elkady, M.; El-Aassar, M.; Hassan, H. Adsorption Profile of Basic Dye onto Novel Fabricated Carboxylated Functionalized Co-Polymer Nanofibers. Polymers 2016, 8, 177. [CrossRef]

3. Liu, C.; Liu, H.; Xu, A.; Tang, K.; Huang, Y.; Lu, C. In situ reduced and assembled three-dimensional graphene aerogel for efficient dye removal. J. Alloys Compd. 2017, 714, 522-529. [CrossRef]

4. Pawar, R.R.; Gupta, P.; Sawant, S.Y.; Shahmoradi, B.; Lee, S.M. Porous synthetic hectorite clay-alginate composite beads for effective adsorption of methylene blue dye from aqueous solution. Int. J. Biol. Macromol. 2018, 114, 1315. [CrossRef] [PubMed]

5. Qian, L.W.; Yang, M.X.; Zhang, S.F.; Hou, C.; Song, W.Q.; Yang, J.F.; Tang, R.H. Preparation of a sustainable bioadsorbent by modifying filter paper with sodium alginate, with enhanced mechanical properties and good adsorption of methylene blue from wastewaters. Cellulose 2018, 25, 2021-2036. [CrossRef]

6. Ai, L.; Li, M.; Li, L. Adsorption of Methylene Blue from Aqueous Solution with Activated Carbon/Cobalt Ferrite/Alginate Composite Beads: Kinetics, Isotherms, and Thermodynamics. J. Chem. Eng. Data 2011, 56, 3475-3483. [CrossRef]

7. Zhu, H.Y.; Fu, Y.Q.; Jiang, R.; Yao, J.; Xiao, L.; Zeng, G.M. Novel magnetic chitosan/poly(vinyl alcohol) hydrogel beads: Preparation, characterization and application for adsorption of dye from aqueous solution. Bioresour. Technol. 2012, 105, 24-30. [CrossRef] [PubMed]

8. Abou, M.T.; Abd, E.M.; Abd, E.R. Radiation preparation of PVA/CMC copolymers and their application in removal of dyes. J. Hazard. Mater. 2009, 168, 68-75.

9. Gong, G.; Zhang, F.; Cheng, Z.; Zhou, L. Facile fabrication of magnetic carboxymethyl starch/poly(vinyl alcohol) composite gel for methylene blue removal. Int. J. Biol. Macromol. 2015, 81, 205-211. [CrossRef] [PubMed]

10. Shao, Z.J.; Huang, X.L.; Yang, F.; Zhao, W.F.; Zhou, X.Z.; Zhao, C.S. Engineering sodium alginate-based cross-linked beads with high removal ability of toxic metal ions and cationic dyes. Carbohydr. Polym. 2018, 187, 85. [CrossRef] [PubMed]

11. Soliman, S.M.A.; Ali, A.M.; Sabaa, M.W. Alginate-based hydrogel for water treatment. Desalination Water Treat. 2017, 94, 129-136. [CrossRef]

12. Zhou, G.; Liu, C.; Chu, L.; Tang, Y.; Luo, S. Rapid and efficient treatment of wastewater with high-concentration heavy metals using a new type of hydrogel-based adsorption process. Bioresour. Technol. 2016, 219, 451-457. [CrossRef] [PubMed]

13. Pourjavadi, A.; Tavakoli, E.; Motamedi, A.; Salimi, H. Facile synthesis of extremely biocompatible double-network hydrogels based on chitosan and poly(vinyl alcohol) with enhanced mechanical properties. J. Appl. Polym. Sci. 2017, 135, 45752. [CrossRef]

14. Ma, J.; Liu, Y.; Ali, O.; Wei, Y.; Zhang, S.; Zhang, Y.; Cai, T.; Liu, C.; Luo, S. Fast Adsorption of Heavy Metal Ions by Waste Cotton Fabrics Based Double Network Hydrogel and Influencing Factors Insight. J. Hazard. Mater. 2017, 344, 1034-1042. [CrossRef]

15. Jiang, X.; Xiang, N.; Zhang, H.; Sun, Y.; Zhen, L.; Hou, L. Preparation and characterization of poly(vinyl alcohol)/sodium alginate hydrogel with high toughness and electric conductivity. Carbohydr. Polym. 2018, 186, 377. [CrossRef] [PubMed]

16. Liu, H.; Liu, C.; Peng, S.; Pan, B.; Lu, C. Effect of polyethyleneimine modified graphene on the mechanical and water vapor barrier properties of methyl cellulose composite films. Carbohydr. Polym. 2018, 182, 52-60. [CrossRef] [PubMed]

17. Liu, H.; Bandyopadhyay, P.; Kshetri, T.; Kim, N.H.; Ku, B.C.; Moon, B.; Lee, J.H. Layer-by-layer assembled polyelectrolyte-decorated graphene multilayer film for hydrogen gas barrier application. Compos. Part $B$ 2017, 114, 339-347. [CrossRef] 
18. Liu, H.; Bandyopadhyay, P.; Kim, N.H.; Moon, B.; Lee, J.H. Surface modified graphene oxide/poly(vinyl alcohol) composite for enhanced hydrogen gas barrier film. Polym. Test. 2016, 50, 49-56. [CrossRef]

19. Liu, H.; Kuila, T.; Kim, N.; Ku, B.C.; Lee, J. In situ synthesis of the reduced graphene oxide-polyethyleneimine composite and its gas barrier properties. J. Mater. Chem. A 2013, 1, 3739-3746. [CrossRef]

20. Yu, Y.; Shu, Y.; Ye, L. In Situ Crosslinking of Poly(vinyl alcohol)/Graphene Oxide-Glutamic Acid Nano-composite Hydrogel as Microbial Carrier: Intercalation Structure and its Wastewater Treatment Performance. Chem. Eng. J. 2018, 336, 306-314. [CrossRef]

21. Liu, S.; Bastola, A.K.; Li, L. A 3D Printable and Mechanically Robust Hydrogel based on Alginate and Graphene Oxide. Acs Appl. Mater. Interfaces 2017, 9, 41473-41481. [CrossRef] [PubMed]

22. Qi, Y.; Yang, M.; Xu, W.; He, S.; Men, Y. Natural polysaccharides-modified graphene oxide for adsorption of organic dyes from aqueous solutions. J. Colloid Interface Sci. 2017, 486, 84-96. [CrossRef] [PubMed]

23. Chen, L.; Li, Y.; Du, Q.; Wang, Z.; Xia, Y.; Yedinak, E.; Lou, J.; Ci, L. High performance agar/graphene oxide composite aerogel for methylene blue removal. Carbohydr. Polym. 2017, 155, 345-353. [CrossRef] [PubMed]

24. Kumar, M.; Tamilarasan, R. Modeling studies: Adsorption of aniline blue by using Prosopis Juliflora carbon/Ca/alginate polymer composite beads. Carbohydr. Polym. 2013, 92, 2171-2180. [CrossRef] [PubMed]

25. Xie, M.; Zhang, F.; Liu, L.; Zhang, Y.; Li, Y.; Li, H.; Xie, J. Surface modification of graphene oxide nanosheets by protamine sulfate/sodium alginate for anti-cancer drug delivery application. Appl. Surf. Sci. 2018, 440, 853-860. [CrossRef]

26. Khalid, I.; Ahmad, M.; Minhas, M.U.; Barkat, K. Preparation and characterization of alginate-PVA-based semi-IPN: Controlled release pH-responsive composites. Polym. Bull. 2018, 75, 1075-1099. [CrossRef]

27. Xiang, S.; Qian, W.; Li, T.; Wang, Y.; Chen, M.; Ma, P.; Dong, W. Hierarchical Structural Double Network Hydrogel with High Strength, Toughness, and Good Recoverability. New J. Chem. 2017, 41, 14397-14402. [CrossRef]

28. Wang, Z.; Zhou, H.; Chen, W.; Li, Q.; Yan, B.; Jin, X.; Ma, A.; Liu, H.; Zhao, W. Dually synergetic network hydrogels with integrated mechanical stretchability, thermal responsiveness and electrical conductivity for strain sensors and temperature alertors. Acs Appl. Mater. Interfaces 2018, 10, 14045-14054. [CrossRef] [PubMed]

29. Fan, J.; Shi, Z.; Lian, M.; Li, H.; Yin, J. Mechanically strong graphene oxide/sodium alginate/polyacrylamide nanocomposite hydrogel with improved dye adsorption capacity. J. Mater. Chem. A 2013, 1, 7433-7443. [CrossRef]

30. Liu, C.; Liu, H.; Lu, C.; Tang, K.; Zhang, Y. Polyethyleneimine-modified graphene oxide/PNIPAm thermoresponsive hydrogels with rapid swelling/deswelling and improved mechanical properties. J. Mater. Sci. 2017, 52, 11715-11724. [CrossRef]

31. Cui, L.; Wang, Y.; Hu, L.; Gao, L.; Du, B.; Wei, Q. Mechanism of Pb(II) and methylene blue adsorption onto magnetic carbonate hydroxyapatite/graphene oxide. Rsc Adv. 2015, 5, 9759-9770. [CrossRef]

32. Dai, H.; Huang, Y.; Huang, H. Eco-friendly polyvinyl alcohol/carboxymethyl cellulose hydrogels reinforced with graphene oxide and bentonite for enhanced adsorption of methylene blue. Carbohydr. Polym. 2017, 185, 1-11. [CrossRef] [PubMed]

33. Liu, C.; Omer, A.M.; Ouyang, X.K. Adsorptive removal of cationic methylene blue dye using carboxymethyl cellulose/k-carrageenan/activated montmorillonite composite beads: Isotherm and kinetic studies. Int. J. Biol. Macromol. 2017, 106, 823-833. [CrossRef] [PubMed]

34. Ghorai, S.; Sarkar, A.; Raoufi, M.; Panda, A.B.; Schönherr, H.; Pal, S. Enhanced removal of methylene blue and methyl violet dyes from aqueous solution using a nanocomposite of hydrolyzed polyacrylamide grafted xanthan gum and incorporated nanosilica. Acs Appl. Mater. Interfaces 2014, 6, 4766. [CrossRef] [PubMed]

35. Yang, X.; Li, Y.; Du, Q.; Sun, J.; Chen, L.; Hu, S.; Wang, Z.; Xia, Y.; Xia, L. Highly effective removal of basic fuchsin from aqueous solutions by anionic polyacrylamide/graphene oxide aerogels. J. Colloid Interface Sci. 2015, 453, 107-114. [CrossRef] [PubMed]

36. Chong, C.; Jie, D.; Bei, L.; Ai, H.; Xiang, Z.; Lang, M.; Shuang, L.; Zhao, C. Toward 3D graphene oxide gels based adsorbents for high-efficient water treatment via the promotion of biopolymers. J. Hazard. Mater. 2013, 263, 467-478. [CrossRef] [PubMed] 
37. Wang, Y.; Feng, Y.; Zhang, X.F.; Zhang, X.; Jiang, J.; Yao, J. Alginate-based attapulgite foams as efficient and recyclable adsorbents for the removal of heavy metals. J. Colloid Interface Sci. 2017, 514, 190. [CrossRef] [PubMed]

38. Melo, B.C.; Faa, P.; Cardoso, V.A.; Agb, P.; Fajardo, A.R.; Fha, R. Cellulose nanowhiskers improve the methylene blue adsorption capacity of chitosan-g-poly(acrylic acid) hydrogel. Carbohydr. Polym. 2018, 181, 358-367. [CrossRef] [PubMed] 